\title{
Effect of time of admission to treatment initiation on outcomes of patients with acute myeloid leukemia: a tertiary care referral center experience
}

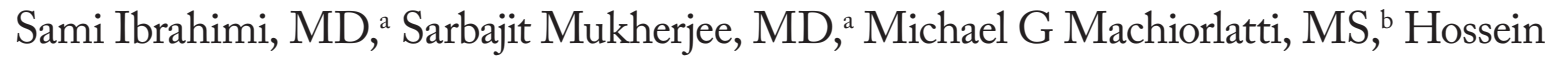

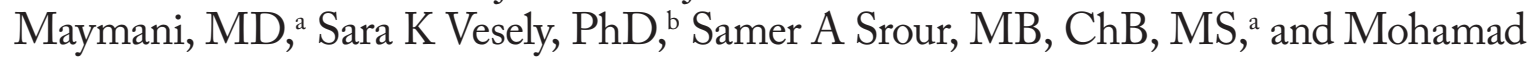 \\ Cherry, $\mathrm{MD}^{\mathrm{a}}$
}

${ }^{a}$ Stephenson Cancer Center and ${ }^{b}$ College of Public Health, the University of Oklahoma Health Sciences Center, Oklahoma City, Oklahoma

Background The time from diagnosis of acute myeloid leukemia (AML) to initiation of treatment could affect patient outcomes, but findings from previous studies have been mixed.

Objective To analyze the impact of the time from admission to treatment initiation (TAT) on overall survival (OS) and event-free survival (EFS) in patients who are newly diagnosed with AML.

Methods A retrospective review of the records of all newly diagnosed AML patients treated at the Oklahoma University Health Sciences Center from January 2000 through June 2015 was conducted. Inclusion criteria also included age $\geq 18$ years and available insurance data. Data on patient characteristics, laboratory values, pathology, treatment, response, and survival were obtained from the electronic medical records.

Results In all, 154 patients were divided into 2 groups: those with a TAT of 0-4 days $(n=109)$ and those with a TAT of $>4$ days $(n=45)$. The median OS of the TAT $0-4$ days group and the TAT $>4$ days group was 1.3 years and 0.57 years, respectively $(P=.0207)$, and the median EFS for the groups was 1.21 years and 0.57 years, respectively $(P=.0392)$. That association remained significant in a multivariate analysis adjusting for age, white blood cell count, molecular risk group, and undergoing allogeneic stem cell transplant.

Limitations Study limitations include a small sample size and a short median follow-up time.

Conclusion Patients with AML who are treated more than 4 days after admission have a lower OS and EFS compared with patients treated within 0-4 days of admission.

Funding/sponsorship None

A cute myeloid leukemia (AML) is the most common acute leukemia in adults in the United States. ${ }^{1}$ In 2018, the estimated annual incidence of AML is 19,520 (32.4\% of all new leukemia cases), with 10,670 projected deaths (43.8\% of all leukemia deaths). ${ }^{1}$ New molecularly targeted treatments are increasingly being used in treating AML, and some of them have shown improved health outcomes. In general, age, white blood cell (WBC) count at presentation, cytogenetics, and molecular characteristics are the major determinants of prognosis and treatment outcome. Studies analyzing the Surveillance Epidemiology and End Results database have also shown racial differences in outcomes. ${ }^{2}$ It is well known to the oncology community that patients with similar characteristics may respond differently to treatment and that outcome is not uniformly related to the well-defined clinical and laboratory characteristics. Issues related to health care disparities and access to health care are also known to affect the outcome in patients with cancer. ${ }^{3-9}$

AML is generally considered by the medical community as a time-sensitive condition. Treatment of patients with AML usually consists of induction chemotherapy followed by consolidation treatment

Accepted for publication September 13, 2018. Correspondence: Sami Ibrahimi, MD; Sami-lbrahimi@ouhsc.edu. Disclosures: Dr Cherry is on the advisory board of Gilead. The remaining authors report no disclosures or conflicts of interest. JCSO 2018;16(5):e 188-e 193. (O2018 Frontline Medical Communications. doi: https://doi.org/10.12788/ ¡cso.0428 


\begin{tabular}{|c|c|c|c|c|}
\hline \multirow[b]{2}{*}{ Variable } & \multirow{2}{*}{$\begin{array}{l}\text { Overall no. } \\
\text { of patients (\%) } \\
(N=154)\end{array}$} & \multicolumn{2}{|c|}{ Time from admission to treatment initiation } & \multirow[b]{2}{*}{$\begin{array}{c}\chi^{2} \\
P \text {-value }\end{array}$} \\
\hline & & $\begin{array}{l}\text { 0-4 days } \\
(\mathrm{n}=109)\end{array}$ & $\begin{array}{l}\text { >4 days } \\
(n=45)\end{array}$ & \\
\hline \multicolumn{5}{|l|}{ Age, y } \\
\hline$<60$ & $107(69.5)$ & $81(74.3)$ & $26(57.8)$ & \multirow[t]{2}{*}{.0427} \\
\hline$\geq 60$ & $47(30.5)$ & $28(25.7)$ & $19(42.2)$ & \\
\hline \multicolumn{5}{|l|}{ Gender } \\
\hline Male & $99(64.3)$ & $67(61.5)$ & $32(71.1)$ & \multirow[t]{2}{*}{.2560} \\
\hline Female & $55(35.7)$ & $42(38.5)$ & $13(28.9)$ & \\
\hline \multicolumn{5}{|l|}{$\begin{array}{l}\text { WBC count at } \\
\text { diagnosis, } \mu / L\end{array}$} \\
\hline$<50 \times 10^{3}$ & $121(78.6)$ & $80(73.4)$ & $41(91.1)$ & \multirow[t]{2}{*}{.0148} \\
\hline$\geq 50 \times 10^{3}$ & $33(21.4)$ & $29(26.6)$ & $4(8.9)$ & \\
\hline \multicolumn{5}{|l|}{ Race } \\
\hline White & $118(77.3)$ & 85 (78.0) & $34(75.6)$ & \multirow{4}{*}{$.0805^{\circ}$} \\
\hline Black & $17(11.0)$ & $10(9.2)$ & $7(15.6)$ & \\
\hline Hispanic & $5(3.3)$ & $2(1.8)$ & $3(6.7)$ & \\
\hline Other & $13(8.4)$ & $12(11.0)$ & $1(2.2)$ & \\
\hline \multicolumn{5}{|l|}{$\begin{array}{l}\text { Cytogenetic/ } \\
\text { molecular risk }\end{array}$} \\
\hline Favorable & $26(16.9)$ & $18(16.5)$ & $8(17.8)$ & \multirow{4}{*}{.6214} \\
\hline Intermediate & $44(28.6)$ & $34(31.2)$ & $10(22.2)$ & \\
\hline Unfavorable & $39(25.3)$ & $25(22.9)$ & $14(31.1)$ & \\
\hline Unknown & $45(29.2)$ & $32(29.4)$ & $13(28.9)$ & \\
\hline \multicolumn{5}{|l|}{ Health insurance } \\
\hline Insured & $123(79.9)$ & $87(79.8)$ & $36(80.0)$ & \multirow[t]{2}{*}{.9794} \\
\hline Uninsured & $31(20.1)$ & $22(20.2)$ & $9(20.0)$ & \\
\hline \multicolumn{5}{|l|}{ AlloSCT ${ }^{b}$} \\
\hline No & $117(77.0)$ & 81 (75.7) & $36(80.0)$ & \multirow[t]{2}{*}{.5655} \\
\hline Yes & $35(23.0)$ & $26(24.3)$ & $9(20.0)$ & \\
\hline \multicolumn{5}{|l|}{ Induction therapy ${ }^{c}$} \\
\hline $7+3$ & $117(79.6)$ & $88(83.8)$ & $29(69.0)$ & \multirow[t]{2}{*}{.0448} \\
\hline Other & $30(20.4)$ & $17(16.2)$ & $13(31)$ & \\
\hline \multicolumn{5}{|l|}{ Day of admission } \\
\hline Monday-Thursday & $115(74.7)$ & 89 (81.7) & $26(57.8)$ & \multirow[t]{2}{*}{.0014} \\
\hline Friday-Sunday & $39(25.3)$ & $20(18.3)$ & $19(42.2)$ & \\
\hline
\end{tabular}

with consideration for stem cell transplant. The duration of time from admission to treatment (TAT) of AML with induction chemotherapy is dependent on multiple factors. These may include the assessment of comorbid conditions and the availability of molecular studies at the time of treatment, which can be time consuming. The effect of treatment delays after AML diagnosis has been investigated, but with conflicting results. One study showed that time from diagnosis to treatment initiation affects survival in younger patients, and another showed it has no effect on survival regardless of patient age. ${ }^{10,11}$ We describe here the results of a retrospective analysis evaluating the impact of 
TABLE 2 Median overall survival and event-free survival (unadjusted) based on TAT and day of admission group ( $\mathrm{N}=154$ )

\begin{tabular}{|c|c|c|c|c|}
\hline \multirow[b]{2}{*}{ Group } & \multicolumn{2}{|c|}{ Overall survival } & \multicolumn{2}{|c|}{ Event-free survival } \\
\hline & Months (95\% CI) & $P$-value & Months (95\% Cl) & P-value \\
\hline 0-4 days TAT & $15.6(9.1-24.1)$ & .0207 & $14.5(8.9-21.1)$ & .0392 \\
\hline$>4$ days TAT & $6.8(4.7-13.8)$ & & $6.8(4.7-12.5)$ & \\
\hline Monday-Thursday & $13.8(8.6-17.8)$ & .9334 & $10.9(8.3-15.6)$ & .9162 \\
\hline Friday-Sunday & $12.5(6.8-21.1)$ & & $9.6(6.8-21.1)$ & \\
\hline
\end{tabular}

TAT, time from admission to treatment initiation

TAT and day of admission on outcomes of patients with AML who received treatment at a tertiary care referral center.

\section{Methods and materials}

We did a retrospective medical record review of all newly diagnosed AML patients at the Oklahoma University Health Sciences Center (OUHSC). Our sample was composed of 154 adult patients. Our inclusion criteria were an age of 18 years or older with complete insurance data, a diagnosis of AML, and having received treatment at our institution from January 2000 through June 2015. Data were obtained on laboratory values at diagnosis, pathology data including cytogenetics, molecular data, and bone marrow biopsies. Data on patient characteristics such as age, race and/or ethnicity, and comorbidities were obtained from the electronic medical records. Treatment data on type and dose of chemotherapy during induction, subsequent treatment phases, and number of treatments to achieve complete response (CR) as well as response data of CR achievement, relapse, date of CR, date of relapse, stem cell transplantation data, date of death, and date of last follow-up visit were recorded retrospectively from the electronic medical record. The study was approved by the OUHSC Institutional Review Board.

\section{Statistical analysis}

TAT was analyzed categorically (0-4 days vs $>4$ days), and day of admission was analyzed categorically (Monday to Thursday vs Friday to Sunday). Descriptive statistics were calculated overall and by TAT group. The chi-square test was used to compare the association between our covariates and TAT. Kaplan-Meier estimates (with a log-rank test) were used to assess the unadjusted effect of TAT with overall survival (OS) and event-free survival (EFS). Median OS and EFS and 95\% confidence intervals (CIs) were also calculated. We used the Cox proportional hazards regression modeling to evaluate the relationship between OS and TAT. The initial model was built by including covariates, with $P<.25$ for the association between the covariates with OS. TAT was maintained in the final model because it was the primary variable of interest, whereas age and risk group were also included in the final model because those covariates are known prognostic risk factors in AML. Among the set of variables screened in, all 2-way interactions were assessed using $P<.05$. No significant interactions were found. Backward elimination was then performed. During the backward elimination, confounding was deemed to have been present if the measure of association of significant variables in the model changed by more than $20 \%$ and the $P$-value of the confounding variable was less than .30. Variables with $P$-values of less than .05 or deemed a confounder would then be retained. A similar modeling approach was used to examine EFS and CR. To evaluate the association between $\mathrm{CR}$ with potential predictors, binary logistic regression was used, whereby day of admission and time to treatment were explored unadjusted and then adjusted for age, WBC count, risk group, and undergoing allogeneic stem cell transplant (AlloSCT). SAS version 9.4 (SAS Institute Inc, Cary, North Carolina) was used for all analyses. A final alpha of 0.05 was used unless otherwise noted.

\section{Results}

Baseline characteristics are presented in Table 1. Treatment was initiated within 4 days for $71 \%$ (109/154) of patients. Most patients in our study were younger than 60 years (70\%), male (64\%), and white (77\%). Most patients were admitted to the hospital for treatment between Monday and Thursday (75\%). A higher proportion of patients in the $0-4$ days TAT group were $<60$ years of age compared with patients in the $>4$ days TAT group $(P=.0427)$. A higher proportion of patients in the 0-4 days TAT group had a WBC count of $\geq 50 \times 10^{3} \mu / \mathrm{L}$ compared with patients in the $>4$ days TAT group ( $27 \%$ vs $9 \%$, respectively; $P=.0148)$. A higher proportion of patients were admitted Friday to Sunday in the TAT $>4$ days group. Insured and uninsured patients were equally distributed between the 2 groups $(P=.0014)$. Cytogenetic and/or molecular risk was not statistically different between the 0-4 days and $>4$ days TAT groups (unfavorable risk, $25 \%$ vs $23 \%$, respectively; $P=.6214)$. A higher proportion of patients received $7+3$ induction chemotherapy ( 7 days cytarabine and 3 days anthracycline) in the TAT 0-4 days group compared with 
the $>4$ days TAT group ( $84 \%$ vs $69 \%$, respectively; $P=.0448)$. The most common intensive chemotherapy regimen used was $7+3(80 \%)$. The rest of the patients $(20 \%)$ received high-dose cytarabine clofarabine-based chemotherapy, hypomethylating agents, or other treatments. The proportion of patients who received an AlloSCT did not differ between the 0-4 days and $>4$ days TAT groups (24\% vs $20 \%$, respectively; $P=.5655$ ).

The median OS for all patients was 10.9 months (95\% CI, 8.3-15.1), and the median EFS was 9.1 months (95\% CI, 7.4-13.8). Median follow-up time was 8.6 months (95\% CI, 6.7-11). We found a significant association between TAT and both OS and EFS without any adjustment (Table 2). The median OS for the TAT 0-4 days group was 15.6 months, and for the TAT $>4$ days group, it was 6.8 months $(P=.0207$; Figure 1$)$. The median EFS for the TAT 0-4 days group was 14.5 months, and for the TAT $>4$ days group, it was 6.8 months $(P=.0240$; Figure 2$)$. We found no association between the day of admission to hospital (Monday-Thursday vs Friday-Sunday) and either OS or EFS. After adjusting for age, WBC count, molecular risk status, and undergoing AlloSCT, the OS was shorter for those who received treatment $>4$ days after admission compared with those who received treatment within 0 to 4 days, with a hazard ratio (HR) of 1.59 (95\% CI, 1.02-2.49; $P=.0427$; Table 3). There was no association between day of admission with OS in the multivariable analysis. Similarly, after adjusting for age, WBC count, molecular risk status, and undergoing AlloSCT, EFS was shorter in patients who received treatment $>4$ days after admission compared with those who received treatment within 0 to 4 days (HR, 1.64; 95\% CI, $1.06-2.54 ; P=.0268)$. There was no association between day of admission with EFS in the multivariable model. Although there was a trend for a higher $\mathrm{CR}$ rate with earlier treatment, this was not statistically significant (Table 4).

\section{Discussion}

Treatment outcomes for patients with AML are known to be affected by several patient- and disease-related factors. Patient-related factors can include age, performance status, comorbidities, and availability of a stem cell donor. Examples of disease-related factors include molecular alterations and site of disease involvement. Little is known about whether the timing of treatment initiation affects patient outcomes. Short-term treatment delays after the diagnosis of leukemia are not uncommon. Generally, patients are treated with anthracycline-based induction

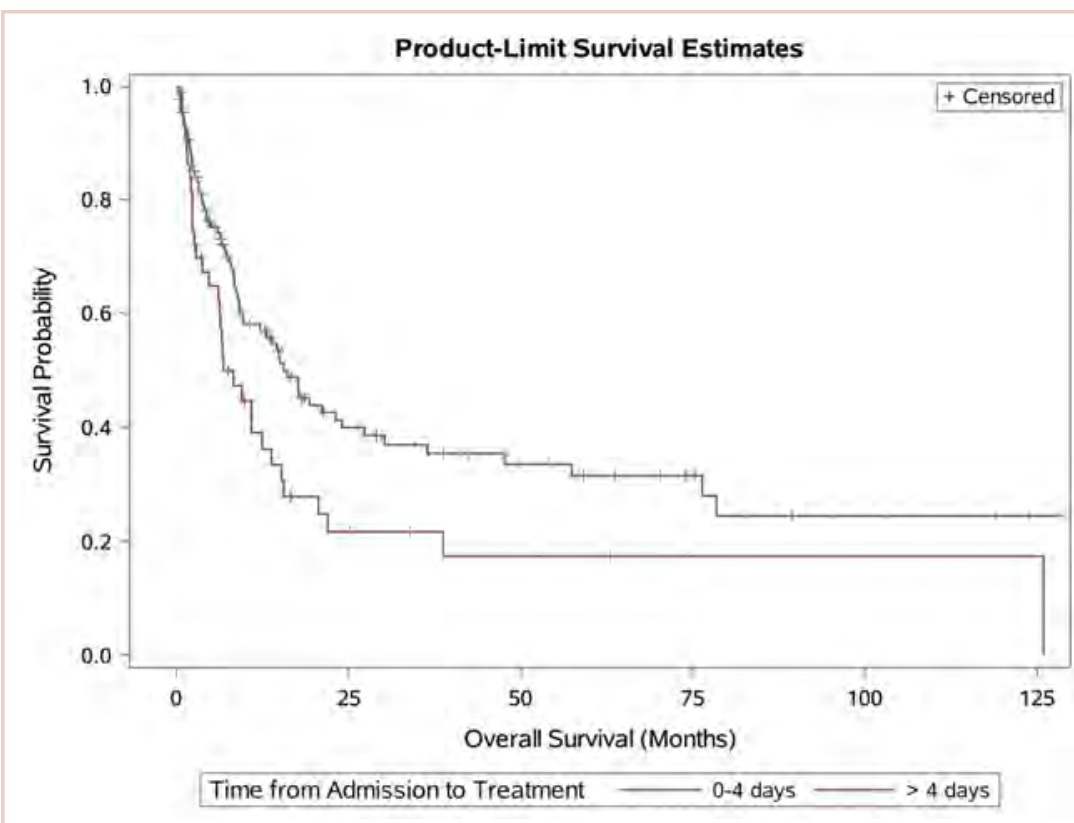

FIGURE 1 Overall survival in months of time to treatment - unadjusted $(P=.0207)$

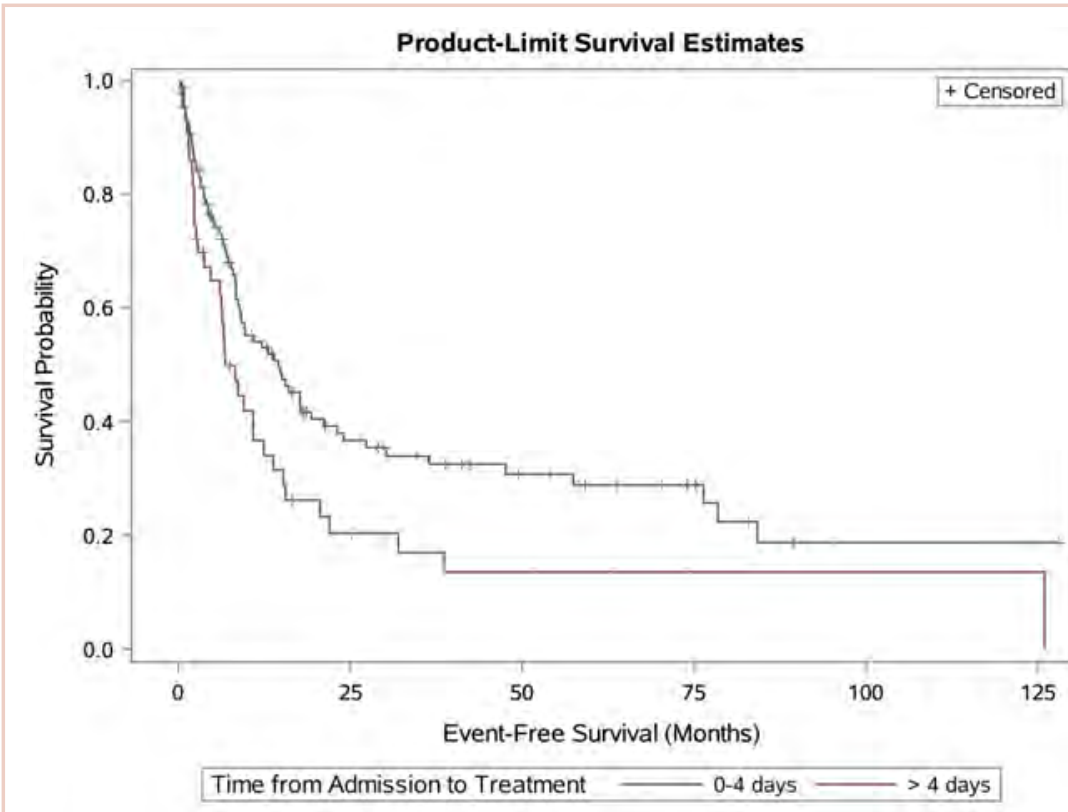

FIGURE 2 Event-free survival in months of time to treatment - unadjusted $(P=.0392)$

chemotherapy, but the response rate and survival are particularly poor in the older age group. ${ }^{12}$ Moreover, increasing comorbidities with aging are expected to lead to lower treatment tolerability. ${ }^{13}$ Therefore, elderly patients are particularly prone to treatment delays while providers await the results of the molecular studies to guide the use of less intensive targeted therapies. ${ }^{10}$ Other reasons for treatment delays may also include transfers between hospitals, sus- 
TABLE 3 TAT adjusted for age, white blood cell count, molecular risk status, and having an AlloSCT ( $N=152,2$ missing AlloSCT)

\begin{tabular}{|c|c|c|c|c|c|}
\hline \multirow[b]{2}{*}{ Parameter } & \multirow[b]{2}{*}{ Group } & \multicolumn{2}{|c|}{ Overall survival } & \multicolumn{2}{|c|}{ Event-free survival } \\
\hline & & HR $(95 \% \mathrm{Cl})$ & $P$-value & HR $(95 \% \mathrm{Cl})$ & $P$-value \\
\hline TAT & $\begin{array}{l}>4 \text { days vs } \\
0-4 \text { days }\end{array}$ & 1.59 (1.02-2.49) & .0427 & $1.64(1.06-2.54)$ & .0268 \\
\hline
\end{tabular}

AlloSCT, allogeneic stem cell transplant; TAT, time from admission to treatment initiation

TABLE 4 Unadjusted and adjusted (age, white blood cell count, risk group, allogeneic stem cell transplant) association between complete response and TAT

$\begin{array}{cccc}\text { Variable } & \text { Group } & \text { OR }(\mathbf{9 5 \%} \text { CI) } & \text { P-value } \\ \text { TAT } & \text { Unadjusted }(n=154) & 0.54(0.27-1.11) & .0944 \\ & \text { Adjusted }(n=152) & 0.67(0.29-1.52) & .3369\end{array}$

TAT, time from admission to treatment initiation

pected or documented infections, and evaluation of chronic illnesses. Our analysis also indicates that admission to the hospital on the weekend contributes to a delay in therapy compared with admission on a weekday.

We found a decreased OS and EFS in patients who received treatment $>4$ days after admission to the hospital compared with patients who received treatment within 0 to 4 days of admission. This association was statistically significant in a bivariate analysis as well as in a multivariable analysis with adjustment for age, WBC count on presentation, molecular risk group, and undergoing AlloSCT. A previous large retrospective study showed that the time from diagnosis to treatment initiation predicts survival in younger, but not older, patients with AML. ${ }^{10}$ This remained true after adjusting for age, performance status, WBC count, and the type of AML in a multivariable analysis. In our study, the declines in overall survival and event-free survival were evident after a delay of more than 4 days.

Another retrospective study that included 599 newly diagnosed AML patients, with a median time from diagnosis to treatment of 8 days, did not show any impact of treatment delay on overall survival, early death, or response rate. ${ }^{11}$ These differences in the effect of treatment delay on outcomes could be related to the differences in baseline characteristics of patients in these studies. Our study had a higher proportion of patients younger than 60 years, for example. We hypothesize that treatment delays, especially in patients with a high WBC count on presentation, might lead to further organ compromise and poorer outcomes with chemotherapy.

In our study, a higher proportion of patients were admitted over the weekend in the $>4$ days TAT group, but when we analyzed the day of admission to hospital separately, it was not associated with OS or EFS. Admission over the weekend was also not associated with clinical outcomes including 30-day mortality in a larger study that included 422 patients treated at a large teaching referral hospital. ${ }^{14}$

Limitations of our study include a small sample size and a short median follow-up time. Most of our patients were young and white, which may not be representative of the general population.

In conclusion, we found that treatment delays are associated with inferior outcomes in AML patients. It remains to be elucidated whether the benefit gained from using targeted and less-intensive chemotherapy, especially in elderly patients, outweighs the potential harm from delaying treatment. Additional studies are needed to confirm our findings in different settings and patient populations.

\section{Acknowledgment}

Statistical support was provided by the Stephenson Cancer Center Biostatistics and Research Design Shared Resource.

\section{References}

1. Siegel RL, Miller KD, Jemal A. Cancer statistics, 2018. CA Cancer J Clin. 2018;68(1):7-30.

2. Patel MI, Ma Y, Mitchell B, Rhoads KF. How do differences in treatment impact racial and ethnic disparities in acute myeloid leukemia? Cancer Epidemiol Biomarkers Prev. 2015;24(2):344-349.

3. Weber JJ, Kachare SD, Vohra NA, Fitzgerald TF, Wong JH. Regional disparities in breast cancer outcomes and the process of care. Am Surg. 2014;80(7):669-674.
4. Shippee TP, Kozhimannil KB, Rowan K, Virnig BA. Health insurance coverage and racial disparities in breast reconstruction after mastectomy. Womens Health Issues. 2014;24(3):e261-e269.

5. Dickens C, Joffe M, Jacobson J, et al. Stage at breast cancer diagnosis and distance from diagnostic hospital in a periurban setting: a South African public hospital case series of over 1,000 women. Int J Cancer. 2014;135(9):2173-2182.

6. Nguyen-Pham S, Leung J, McLaughlin D. Disparities in breast can- 
cer stage at diagnosis in urban and rural adult women: a systematic review and meta-analysis. Ann Epidemiol. 2014;24(3):228-235.

7. Gong G, Belasco E, Hargrave KA, Lyford CP, Philips BU Jr.

Determinants of delayed detection of cancers in Texas counties in the United States of America. Int J Equity Health. 2012;11:29.

8. Erhunmwunsee L, Joshi MB, Conlon DH, Harpole DH Jr.

Neighborhood-level socioeconomic determinants impact outcomes in nonsmall cell lung cancer patients in the Southeastern United States. Cancer. 2012;118(20):5117-5123.

9. Steele CB, Pisu M, Richardson LC. Urban/rural patterns in receipt of treatment for non-small cell lung cancer among black and white Medicare beneficiaries, 2000-2003. J Natl Med Assoc. 2011;103(8):711-718.

10. Sekeres MA, Elson P, Kalaycio ME, et al. Time from diagnosis to treatment initiation predicts survival in younger, but not older, acute myeloid leukemia patients. Blood. 2009;113(1):28-36.
11. Bertoli S, Bérard E, Huguet F, et al. Time from diagnosis to intensive chemotherapy initiation does not adversely impact the outcome of patients with acute myeloid leukemia. Blood. 2013:121(14):2618-2626.

12. Shah A, Andersson TM, Rachet B, Björkholm M, Lambert PC. Survival and cure of acute myeloid leukaemia in England, 1971-2006: a population-based study. Br J Haematol. 2013;162(4):509-516.

13. Mohammadi M, Cao Y, Glimelius I, Bottai M, Eloranta S, Smedby $\mathrm{KE}$. The impact of comorbid disease history on all-cause and cancerspecific mortality in myeloid leukemia and myeloma - a Swedish population-based study. BMC Cancer. 2015;15:850.

14. Bejanyan N, Fu AZ, Lazaryan A, et al. Impact of weekend admissions on quality of care and outcomes in patients with acute myeloid leukemia. Cancer. 2010;116(15):3614-3620. 